\title{
Bermuda's Walsingham Caves: A Global Hotspot for Anchialine Stygobionts
}

\author{
Thomas M. Iliffe *(i) and Fernando Calderón-Gutiérrez (iD \\ Department of Marine Biology, Texas A\&M University at Galveston, Galveston, TX 77553-1675, USA; \\ fercg12@tamu.edu \\ * Correspondence: iliffet@tamug.edu
}

Citation: Iliffe, T.M.;

Calderón-Gutiérrez, F. Bermuda's Walsingham Caves: A Global Hotspo for Anchialine Stygobionts. Diversity 2021, 13, 352. https://doi.org/10. $3390 / \mathrm{d} 13080352$

Academic Editors: Tanja Pipan, David C. Culver and Louis Deharveng

Received: 10 June 2021

Accepted: 9 July 2021

Published: 30 July 2021

Publisher's Note: MDPI stays neutral with regard to jurisdictional claims in published maps and institutional affiliations.

Copyright: (C) 2021 by the authors Licensee MDPI, Basel, Switzerland. This article is an open access article distributed under the terms and conditions of the Creative Commons Attribution (CC BY) license (https:// creativecommons.org/licenses/by/ $4.0 /)$.

\begin{abstract}
Bermuda is an Eocene age volcanic island in the western North Atlantic, entirely capped by Pleistocene eolian limestone. The oldest and most highly karstified limestone is a $2 \mathrm{~km}^{2}$ outcrop of the Walsingham Formation containing most of the island's 150+ caves. Extensive networks of submerged cave passageways, flooded by saltwater, extend under the island. In the early 1980s, cave divers initially discovered an exceptionally rich and diverse anchialine community inhabiting deeper sections of the caves. The fauna inhabiting caves in the Walsingham Tract consists of 78 described species of cave-dwelling invertebrates, including 63 stygobionts and 15 stygophiles. Thus, it represents one of the world's top hotspots of subterranean biodiversity. Of the anchialine fauna, 65 of the 78 species are endemic to Bermuda, while 66 of the 78 are crustaceans. The majority of the cave species are limited in their distribution to just one or only a few adjacent caves. Due to Bermuda's high population density, water pollution, construction, limestone quarries, and trash dumping produce severe pressures on cave fauna and groundwater health. Consequently, the IUCN Red List includes 25 of Bermuda's stygobiont species as critically endangered.
\end{abstract}

Keywords: biodiversity; fauna; conservation; seamount; ecology

\section{Introduction}

Bermuda is a small, mid-Atlantic island located $1050 \mathrm{~km}$ off the east coast of the United States in the Sargasso Sea, at $32^{\circ} 20^{\prime} \mathrm{N}, 64^{\circ} 45^{\prime} \mathrm{W}$. It lies approximately equidistant by air from Boston, New York, and Atlanta, making Bermuda only a short flight away for all of the U.S. east coast. Due to its warm climate, clear tropical waters, coral reefs, pink sand beaches, and ease of access, Bermuda is a popular tourist destination.

Although the Spanish explorer Juan de Bermudez discovered these isolated islands in 1505, he did not try to land. Ten years later, he returned to Bermuda, leaving behind a dozen pigs and sows for any castaways who might become stranded there. In 1609, the English sailing ship Sea Venture, on its way to resupply the Jamestown Colony, was caught in a strong storm and wrecked on Bermuda's reefs. The survivors of the shipwreck were stranded on the previously uninhabited island for nine months until two new ships could be constructed from local timber. The settlement of Bermuda did not occur until 1612, when the town of St. George officially became Bermuda's first capital and the oldest continually inhabited English town in the New World [1].

Bermuda's caves have been long recognized and prominently mentioned in early written works on the island [2]. Shakespeare's play, The Tempest, was likely inspired by the Bermuda shipwreck and takes place in and around a cave. The first published reference to Bermuda caves was in 1623, when Captain John Smith (of Pocahontas fame) described, "in some places varye strange, darke, and cumbersome Caues." John Hardy's 1671 poetic description of Bermuda caves states [3]: 
"The water flowing to them [Harrington Sound] underground,

Being most salt, and all along the shore

There are dark caves, of a miles length or more

Extending under ground, in which there be

Deep holes with water, though no one can see

A passage for it in ..."

The 1872-1876 round-the-world voyage of H.M.S. Challenger, which marked the beginning of modern oceanography, included a stopover in Bermuda. The expedition's commander, Captain George Nares, took the opportunity to explore Paynter's Vale (a.k.a. Church) Cave, accompanied by the British Governor of Bermuda, Sir John Henry Lefroy. Nares rowed the governor across a lake at the bottom of the cave, remarking on "a slight change of level with the tide, sufficient to keep the water perfectly pure" [4].

\section{Anchialine}

Since Bermuda is a relatively small island with no place very far from, or very high above the sea, caves that descend deep enough, end at very clear, exceptionally blue seawater pools. However, until the beginning of cave diving in the early 1980s, Bermuda's underwater caves were unexplored and unknown, as were the animals living in them. When cave divers discovered an amazing variety of new species, new genera, and even several new orders, Bermuda's saltwater caves clearly merited status as a unique ecosystem [5].

After examining an assortment of highly unusual caridean shrimps from tropical land-locked saltwater pools on the several Indo-West Pacific islands and from the Sinai Peninsula, Dutch carcinologist, L.B. Holthuis, recognized the significance of this habitat. He created the term "anchialine" to describe "pools with no surface connection to the sea, containing salt or brackish water, which fluctuates with the tides" [6]. At the 1984 International Symposium on the Biology of Marine Cave held in Bermuda, Holthuis's original definition was expanded and modified to include tidal, saltwater pools inside caves: "Anchialine habitats consist of bodies of haline water, usually with a restricted exposure to open air, always with more or less extensive subterranean connections to the sea, and showing noticeable marine as well as terrestrial influences" [7]. During the 2012 Second International Symposium on Anchialine Ecosystems in Croatia, the term anchialine was more broadly defined as "a tidally-influenced subterranean estuary located within crevicular and cavernous karst and volcanic terrains that extends inland to the limit of seawater penetration" [8]. The "anchialine habitat continuum", as described by van Hengstum et al. (2019), extends uninterrupted from as far inland as saline groundwater penetrates, to the offshore edge of the platform shelf. In the Pleistocene, sea level changes alternately exposed and flooded the caves, such that anchialine groundwater alternately regressed or flooded bedrock voids [9].

\section{Bermuda Geography and Geology}

The islands of Bermuda lie atop the Bermuda Seamount, a volcanic peak rising from a seafloor depth of more than $4000 \mathrm{~m}$. A ring of coral reefs surround a central lagoon on the flattened summit of this long extinct volcano. The islands of Bermuda, on the southeast edge of the platform, enclose several harbors and small bays. Eolian limestone completely caps the Bermuda Seamount to form the island mass. Limestone stratigraphy shows numerous cycles of subaerial eolianite and shallow marine carbonate deposition during interglacial high sea stands alternating with red clay soil horizons, marking glacial episodes of lowered sea level. Eolianites represented as lithified sand dune ridges, constitute more than $90 \%$ of the limestone volume. The main eolianite units are separated by fossils soils and, listed in order of increasing age, are the Southampton, Rocky Bay, Belmont, Lower Town Hill, Upper Town Hill, and Walsingham Formations. The Walsingham Formation, made up of highly altered and very dense eolianite, was deposited $\geq 700,000$ years ago [10]. 


\section{Bermuda Caves}

Bermuda caves are believed to have formed syngenetically by a phreatic solution of limestone during glacial low stands of sea level. When the Ice Age sea level was down, the top of the seamount was emergent, and the islands' total land mass was about 13 times as large as it is today. As a result, the sizable body of fresh groundwater necessary for cave formation was present. As post-glacial sea levels rose, large portions of the caves were drowned by the encroaching seawater as it displaced the freshwater. Continuing collapse of overlying rock into the large voids created the irregular chambers and fissure entrances characteristic of Bermuda's caves [11].

Most of Bermuda's 150+ known caves are located in the Walsingham Tract, a $4 \mathrm{~km}$ long by $0.5 \mathrm{~km}$ wide surface outcropping of the Walsingham Formation, situated between Harrington Sound and Castle Harbour (Figure 1A,B). The Walsingham Tract contains the island's longest dry caves, most notably Church, Wonderland, Admiral's, Sibley's, and Jane's Caves. Crystal, Wonderland, Walsingham, Palm Caves, and others, are isolated cave entrances, interconnected as segments of a large hydrologically linked cave system. The underwater portions of caves in the Walsingham Tract reach water depths of $24 \mathrm{~m}$, but still closely resemble their dry upper levels, even to the variety of large, subaerial-formed speleothems found above and below water. Caves in the Shelly Bay area, on the opposite side of Harrington Sound, are exclusively underwater with practically no dry portions. These caves have long, nearly level, anastomosing underwater passages at $18 \mathrm{~m}$ depth, with entrances on Harrington Sound and passageway extending under the island. Bermuda's longest cave, the $2 \mathrm{~km}$ long Green Bay Cave System, is located here [9,12].

Harrington Sound is an almost totally enclosed body of saltwater, with its only connection to the sea being a narrow channel at Flatt's Inlet (Figure 1B). Due to its restricted access to the ocean, tides in Harrington Sound have only 25\% the range of ocean tides and occur $3 \mathrm{~h}$ later. When the volume of water tidally exchanging through Flatt's Inlet is compared with the total tidal volume of Harrington Sound (area X tidal range), only about half of the tidal volume passes out through the inlet, while the remainder moves through submerged caves [13]. Tidal exchange primarily occurs in caves around the periphery of Harrington Sound where the land is the narrowest. On the east side of the Sound, exchange occurs through caves from the Walsingham Tract (Walsingham and Palm Cave Systems) to Castle Harbor, on the west side through Shelly Bay (Green Bay and Red Bay Caves) to the North Shore, and on the south side (Devil's Hole Caves) to Bermuda's South Shore. Cave pools in the Walsingham and Palm cave systems have tide ranges that decrease, while residence times increase with their relative distance away from Castle Harbour and approaching Harrington Sound. The Crystal and Wonderland Cave sections of the Walsingham System (Figure 1C) are off the main flow channels where circulation patterns are restricted. Their residence times are much longer, with the phreatic zone consisting of very clear, slowly moving or stagnant waters with a lower surface salinity [14]. Caves with greater water transport, e.g., Palm Cave System (Figure 1D), have surface waters only slightly diluted and reach normal marine salinity (35-36 ppt) below $1 \mathrm{~m}$ depths. The food input into caves is primarily plankton and organic matter derived from the sea itself, although primary production in open anchialine pools may provide an additional source of food in the submerged caves (Figure 2A) [15]. 


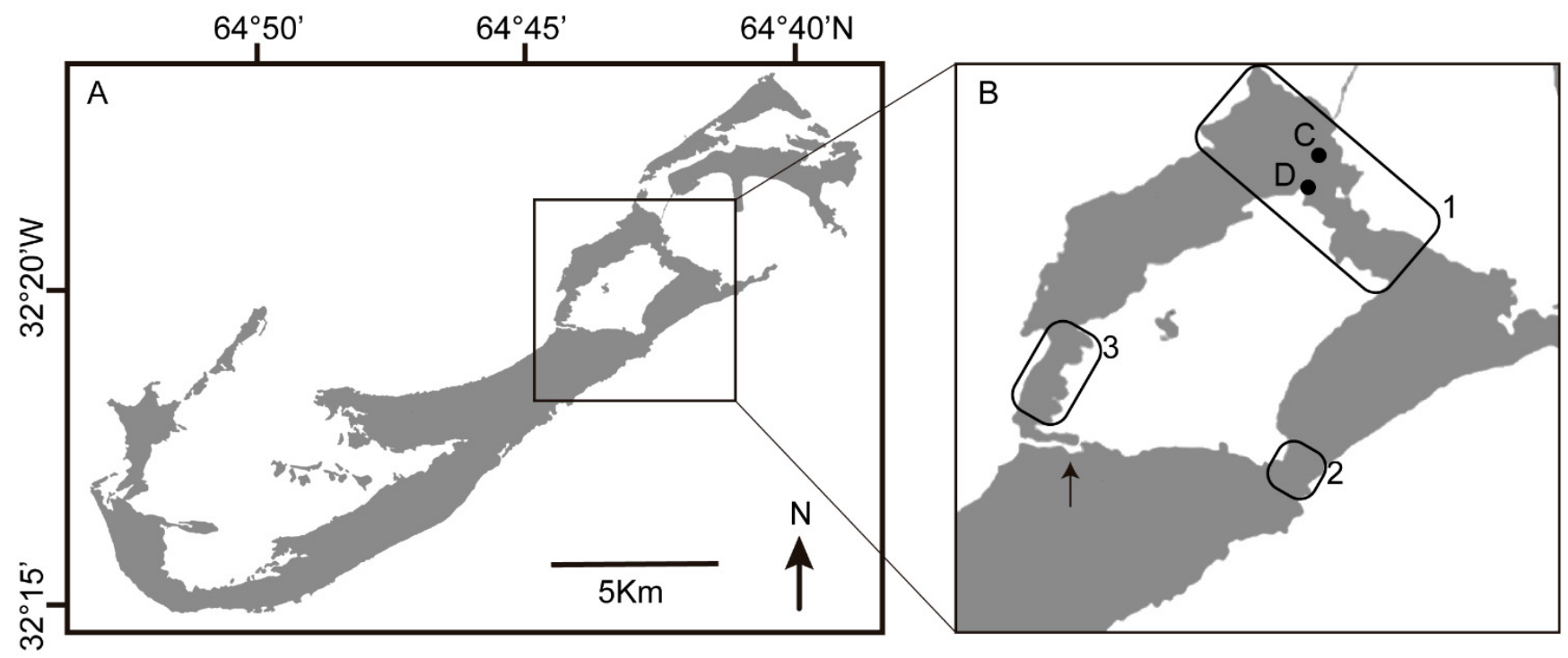

C

D

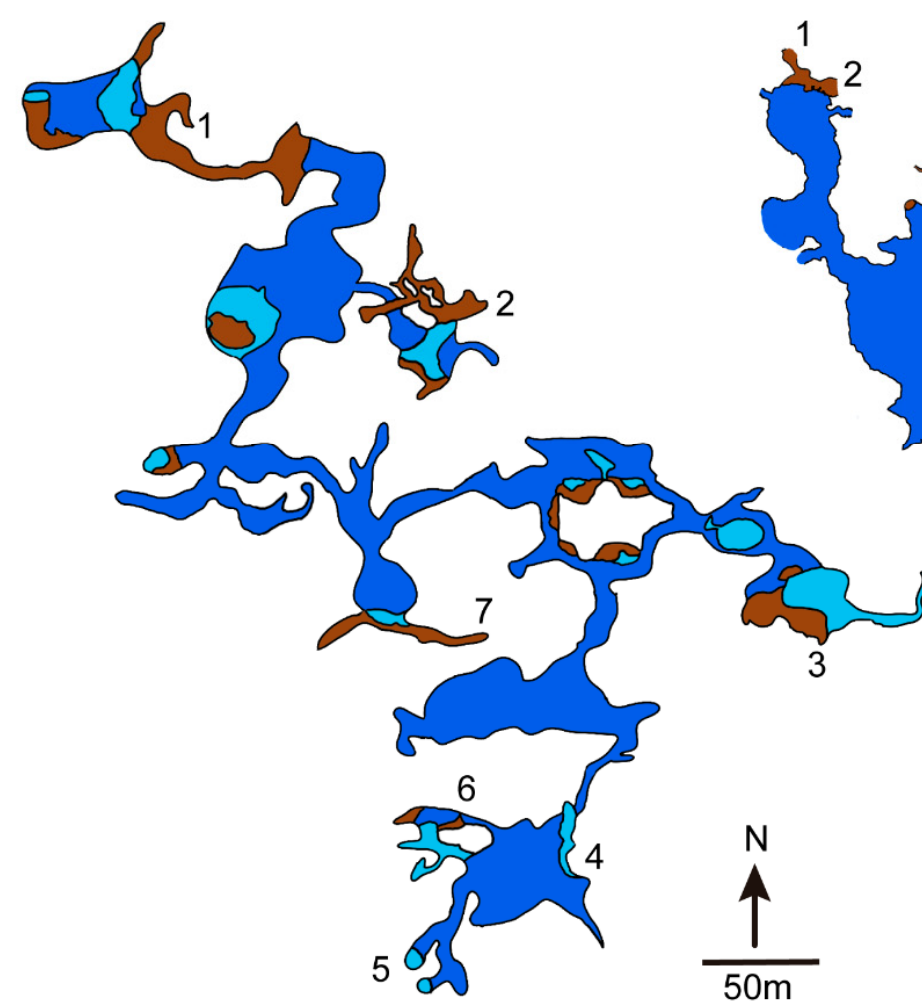

Dry cave

Pool or lake

Submerged cave

Figure 1. (A) Map of Bermuda in the North Atlantic Ocean, (B) Cave regions surrounding Harrington Sound, a large inland body of water mostly encircled by land. The arrow indicates the only opening to the sea at Flatt's Inlet, rectangles show regions of cave and karst development in (1) the Walsingham Tract (with the letters C and D indicating the location of the two main caves), (2) Devil's Hole, (3) Shelly Bay, (C) Walsingham Cave System with cave entrances: (1) Wonderland, (2) Crystal, (3) Walsingham, (4) Deep Blue, (5) Vine, (6) Old Horse, (7) Fern Sink (modified from map by Robert Power), (D) Palm Cave System with cave entrances: (1) Palm Slit, (2) Palm, (3) Strawmarket, (4) Sailor's Choice, (5) Myrtle Bank, (6) Cripple Gate (modified from map by Jason Richards). 


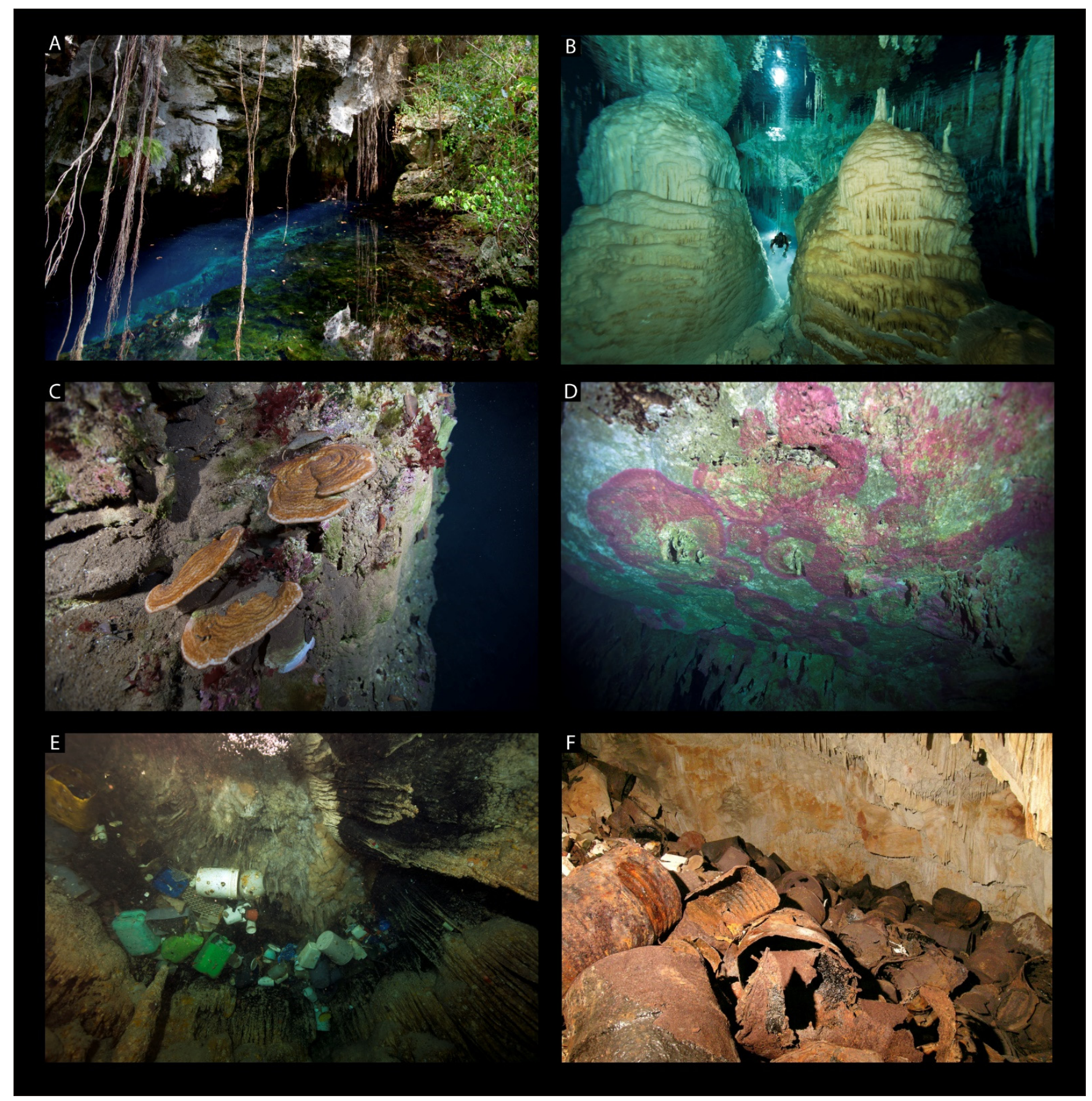

Figure 2. Caves of the Walsingham Tract. (A) Deep Blue Cave entrance pool, (B) Cave diver between underwater stalagmites near the Crystal Cave, (C) Fragile saucer coral (Agarcia fragilis Dana, 1848) in the cavern pool of Deep Blue, (D) Coraline algae in the cavern ceiling of Deep Blue, (E) Submerged plastic garbage floating on the ceiling in the Palm Cave System, and (F) Rusting and disintegrating bitumen barrels in Bitumen Cave (in the Walsingham Tract). Photo credits: Tamara Thomsen (A); Jill Heinerth (B,E). 


\section{Cave Biology}

Open water marine habitats in Bermuda have been well investigated, including numerous studies focusing on lightless environments in the deep sea and interstitial. The Bermuda Biological Station for Research (now the Bermuda Institute of Ocean Sciences) has continuously conducted marine biological research since 1903. The Bermuda Aquarium, Museum and Zoo, founded in 1926, focuses on oceanic island species and the conservation, education, and research related to them. In contrast, serious biological investigations of the underwater caves did not begin until the advent of cave diving [16].

Since Bermuda's terrestrial caves contain little organic matter, there are no known endemic terrestrial species and, in general, the fauna is sparse. Cave-dwelling bats, or other animals that bring organic material into the caves, are lacking in Bermuda. However, a rich and diverse marine biota inhabits the submarine passageways and anchialine pools of Bermuda's caves. Although many marine species are accidental or occur only at coastal cave entrances, a variety of stygobiont (i.e., aquatic cave-adapted) taxa are present, with the Walsingham Cave System - containing the greatest number of Bermuda stygobiontsincluded as one of the original subterranean biodiversity hotspots [17].

Anchialine cave fauna have the same adaptations to caves as freshwater and terrestrial cave organisms, i.e., reduction or loss of eyes and pigmentation, elongation of appendages, and increase in nonvisual sensory receptors [18]. Recent cave colonists tend to have pigmented body and eyes, while ancient settlers of caves have lost their eyes and pigment. The eyes of Bermuda's anchialine fauna show varying degrees of adaptations to the cave environment, ranging from the depigmented and eyeless Mictocaris halope, intermediate Typhlatya iliffei with small eyes and little pigment, to Parhippolyte sterreri having large eyes and bright red pigmentation (Figure 3) [19-21].

Seventy-eight cave-adapted species have been recorded in caves from the Walsingham Tract, Bermuda. The great majority of these species are crustaceans, making up $85 \%$ of the fauna: 20 copepods, 19 species of ostracods, 7 amphipods, 5 cumaceans and shrimps, 4 isopods, 2 mysids and tanaidaceans, and 1 mictacean and ingolfiellid. Non-crustacean species include five mites, three polychaetes, and two ciliates and mollusks. The poor representation of taxa other than crustaceans could be due to an effort or specialist bias [22]. Ten of the recorded species are the only representative of their genus, while Mictocaris halope is the only known species of its order. Twenty species belong to exclusively stygobiont taxa, while eighteen species have close relatives inhabiting the deep-sea (Table 1, Figure 3). 
A

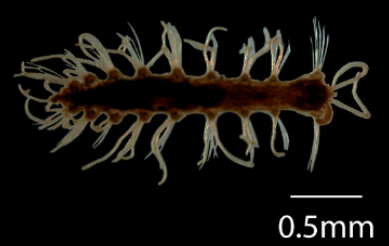

D

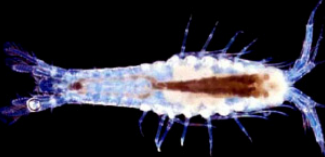

$\overline{1 \mathrm{~mm}}$
B

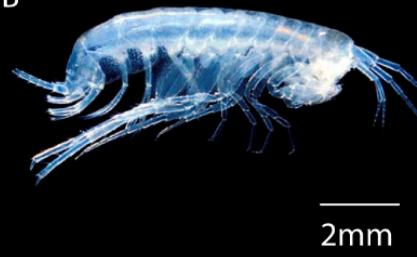

$2 \mathrm{~mm}$
G

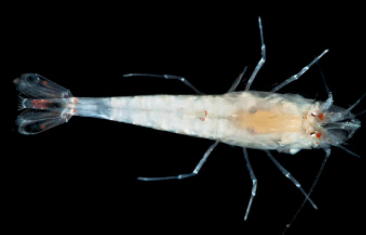

E

H

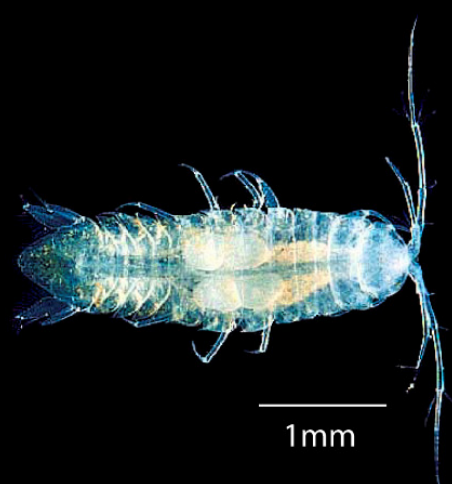

F

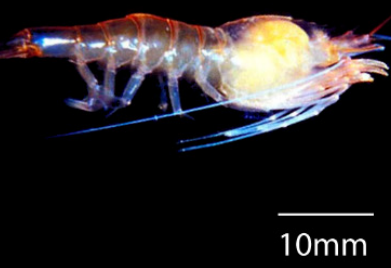

$0 \mathrm{~mm}$

I

C
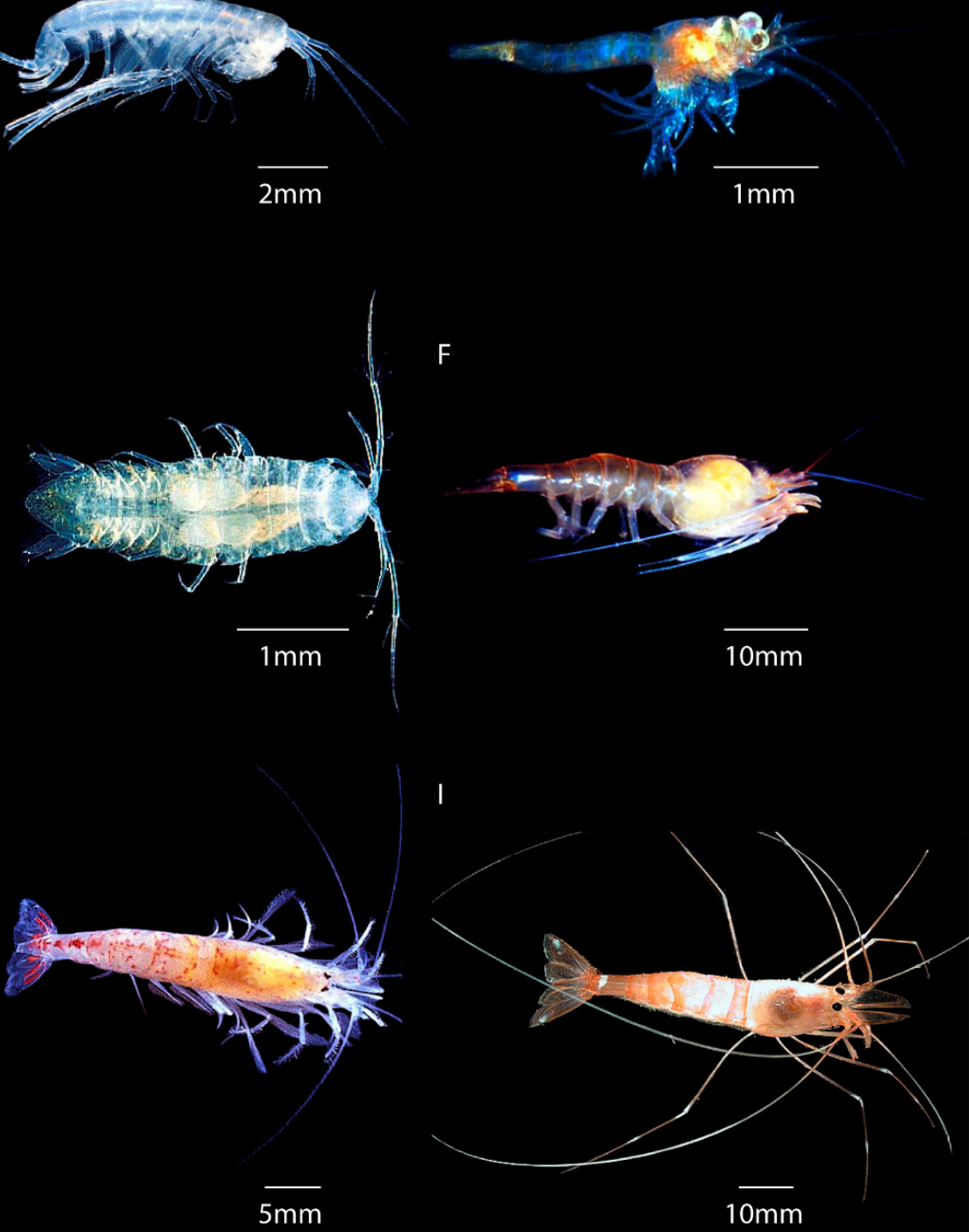

Figure 3. Examples of stygobiont species from the Walsingham cave system. (A) Leptonerilla prospera, (B) Pseudoniphargus grandimanus, (C) Bermudamysis speluncola, (D) Mictocaris halope, (E) Arubolana aruboides, (F) Barbouria cubensis, (G) Typhlatya iliffei, (H) Procaris chacei, and (I) Parhippolyte sterreri. Photo credits: (A) from Katrine Worsaae. 


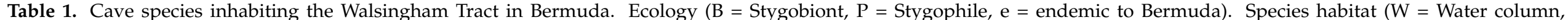

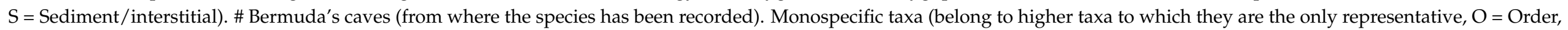

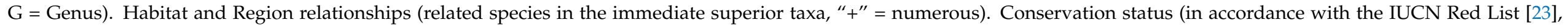
$\mathrm{Cr}=$ Critically Endangered). Taxonomic status was validated in WoRMS [24].

\begin{tabular}{|c|c|c|c|c|c|c|c|c|c|c|c|c|}
\hline & \multirow{2}{*}{ Ecology } & \multirow{2}{*}{$\begin{array}{l}\text { Species } \\
\text { Habitat }\end{array}$} & \multirow{2}{*}{$\begin{array}{l}\text { \# Bermuda } \\
\text { Caves }\end{array}$} & \multirow{2}{*}{$\begin{array}{l}\text { Monospecific } \\
\text { Taxa }\end{array}$} & \multirow{2}{*}{$\begin{array}{l}\text { Species in } \\
\text { the Genus }\end{array}$} & \multicolumn{2}{|c|}{ Habitat Relationship } & \multicolumn{3}{|c|}{ Region Relationship } & \multirow{2}{*}{$\begin{array}{l}\text { Conservation } \\
\text { Status }\end{array}$} & \multirow{2}{*}{ Reference } \\
\hline & & & & & & Cave/Interstitial & Deep Sea & Caribbean & Atlantic & Other & & \\
\hline \multicolumn{13}{|l|}{ Chromista } \\
\hline \multicolumn{13}{|l|}{ Ciliophora, Euplotidae } \\
\hline $\begin{array}{l}\text { Euplotes iliffei Hill \& Small in } \\
\text { Hill, Small \& Iliffe, } 1986\end{array}$ & $\mathrm{~B}, \mathrm{e}$ & $\mathrm{W}$ & 1 & & 50 & & & $x$ & $x$ & $x$ & & [25] \\
\hline \multicolumn{13}{|l|}{$\begin{array}{c}\text { Ciliophora, } \\
\text { Parauronematidae }\end{array}$} \\
\hline $\begin{array}{l}\text { Glauconema bermudense } \\
\text { Small, } 1986\end{array}$ & $\mathrm{~B}, \mathrm{e}$ & $\mathrm{W}$ & 4 & & 2 & & & & $x$ & & & [26] \\
\hline \multicolumn{13}{|l|}{ Annelida } \\
\hline \multicolumn{13}{|l|}{ Polychaeta, Nerillidae } \\
\hline $\begin{array}{c}\text { Leptonerilla prospera (Sterrer \& } \\
\text { Iliffe, 1982) }\end{array}$ & $\mathrm{B}, \mathrm{e}$ & $\mathrm{S}$ & 8 & & 3 & 2 & & & $x$ & $x$ & $\mathrm{Cr}$ & [27] \\
\hline $\begin{array}{l}\text { Speleonerilla saltatrix (Worsaae, } \\
\text { Sterrer \& Iliffe, 2004) }\end{array}$ & $\mathrm{B}, \mathrm{e}$ & S & 1 & & 4 & 3 & & $x$ & $x$ & & & [28] \\
\hline \multicolumn{13}{|l|}{ Oligochaeta, Tubificidae } \\
\hline \multicolumn{13}{|l|}{ Mollusca } \\
\hline \multicolumn{13}{|l|}{ Gastropoda, Caecidae } \\
\hline $\begin{array}{l}\text { Caecum caverna Moolenbeek, } \\
\text { Faber \& Iliffe, } 1988\end{array}$ & $\mathrm{~B}, \mathrm{e}$ & $\mathrm{S}$ & 2 & & 218 & 1 & & $x$ & $x$ & $x$ & & [30] \\
\hline $\begin{array}{c}\text { Caecum troglodyta } \\
\text { Moolenbeek, Faber \& } \\
\text { Iliffe, } 1988\end{array}$ & $\mathrm{~B}, \mathrm{e}$ & S & 2 & & 218 & 1 & & $x$ & $x$ & $x$ & & [30] \\
\hline
\end{tabular}


Table 1. Cont.

\begin{tabular}{|c|c|c|c|c|c|c|c|c|c|c|c|c|}
\hline & \multirow{2}{*}{ Ecology } & \multirow{2}{*}{$\begin{array}{l}\text { Species } \\
\text { Habitat }\end{array}$} & \multirow{2}{*}{$\begin{array}{l}\text { \# Bermuda } \\
\text { Caves }\end{array}$} & \multirow{2}{*}{$\begin{array}{l}\text { Monospecific } \\
\text { Taxa }\end{array}$} & \multirow{2}{*}{$\begin{array}{l}\text { Species in } \\
\text { the Genus }\end{array}$} & \multicolumn{2}{|c|}{ Habitat Relationship } & \multicolumn{3}{|c|}{ Region Relationship } & \multirow{2}{*}{$\begin{array}{l}\text { Conservation } \\
\text { Status }\end{array}$} & \multirow{2}{*}{ Reference } \\
\hline & & & & & & Cave/Interstitial & Deep Sea & Caribbean & Atlantic & Other & & \\
\hline \multicolumn{13}{|l|}{ Arthropoda } \\
\hline \multicolumn{13}{|l|}{ Arachnida } \\
\hline \multicolumn{13}{|l|}{ Acari, Halacaridae } \\
\hline $\begin{array}{c}\text { Agauopsis bermudensis Bartsch } \\
\text { \& Iliffe, } 1985\end{array}$ & $\mathrm{~B}, \mathrm{e}$ & $\mathrm{S}$ & 2 & & 90 & 1 & & $x$ & $x$ & $x$ & & {$[31]$} \\
\hline $\begin{array}{l}\text { Agauopsis littoralis Bartsch \& } \\
\text { Iliffe, } 1985\end{array}$ & $\mathrm{~B}, \mathrm{e}$ & S & 1 & & 90 & 1 & & $x$ & $X$ & $x$ & & {$[31]$} \\
\hline $\begin{array}{l}\text { Copidognathus bermudensis } \\
\text { Bartsch \& Iliffe, } 1985\end{array}$ & $\mathrm{~B}, \mathrm{e}$ & S & 2 & & 380 & 2 & & $x$ & $x$ & $x$ & & [31] \\
\hline $\begin{array}{l}\text { Copidognathus longispinus } \\
\text { Bartsch \& Iliffe, } 1985\end{array}$ & $\mathrm{~B}, \mathrm{e}$ & S & 1 & & 380 & 2 & & $x$ & $x$ & $x$ & & {$[31]$} \\
\hline $\begin{array}{l}\text { Copidognathus subterraneus } \\
\text { Bartsch \& Iliffe, } 1985\end{array}$ & $\mathrm{~B}, \mathrm{e}$ & S & 1 & & 380 & 2 & & $x$ & $x$ & $x$ & & [31] \\
\hline \multicolumn{13}{|l|}{ Crustacea } \\
\hline \multicolumn{13}{|l|}{ Copepoda, Calanoida } \\
\hline $\begin{array}{l}\text { Epacteriscus rapax } \\
\text { Fosshagen, } 1973\end{array}$ & $\mathrm{P}$ & W & 9 & & 3 & 2 & & & $x$ & $x$ & & [33] \\
\hline $\begin{array}{c}\text { Erebonectes nesioticus } \\
\text { Fosshagen, in Fosshagen \& } \\
\text { Iliffe, } 1985\end{array}$ & $\mathrm{~B}, \mathrm{e}$ & W & 4 & G & 1 & 29 & 1 & $x$ & $X$ & & $\mathrm{Cr}$ & [34] \\
\hline $\begin{array}{l}\text { Exumella polyarthra } \\
\text { Fosshagen, } 1970\end{array}$ & $\mathrm{P}$ & $\mathrm{W}$ & $x$ & & 4 & 2 & & $x$ & & $x$ & & [35] \\
\hline $\begin{array}{l}\text { Miostephos leamingtonensis } \\
\text { Yeatman, } 1980\end{array}$ & $\mathrm{~B}, \mathrm{e}$ & W & 1 & & 2 & 1 & & $x$ & & & & [36] \\
\hline $\begin{array}{l}\text { Paracyclopia naessi Fosshagen } \\
\text { in Fosshagen \& Iliffe, } 1985\end{array}$ & $\mathrm{~B}, \mathrm{e}$ & $\mathrm{W}$ & 5 & G & 1 & 4 & & & & & $\mathrm{Cr}$ & [34] \\
\hline $\begin{array}{l}\text { Ridgewayia marki } \\
\text { (Esterly, 1911) }\end{array}$ & $\mathrm{P}$ & W & 2 & & 14 & 4 & & $X$ & $X$ & $X$ & & [37] \\
\hline
\end{tabular}


Table 1. Cont.

\begin{tabular}{|c|c|c|c|c|c|c|c|c|c|c|c|c|}
\hline & \multirow{2}{*}{ Ecology } & \multirow{2}{*}{$\begin{array}{l}\text { Species } \\
\text { Habitat }\end{array}$} & \multirow{2}{*}{$\begin{array}{l}\text { \# Bermuda } \\
\text { Caves }\end{array}$} & \multirow{2}{*}{$\begin{array}{l}\text { Monospecific } \\
\text { Taxa }\end{array}$} & \multirow{2}{*}{$\begin{array}{l}\text { Species in } \\
\text { the Genus }\end{array}$} & \multicolumn{2}{|c|}{ Habitat Relationship } & \multicolumn{3}{|c|}{ Region Relationship } & \multirow{2}{*}{$\begin{array}{l}\text { Conservation } \\
\text { Status }\end{array}$} & \multirow{2}{*}{ Reference } \\
\hline & & & & & & Cave/Interstitial & Deep Sea & Caribbean & Atlantic & Other & & \\
\hline \multicolumn{13}{|l|}{ Copepoda, Cyclopoida } \\
\hline $\begin{array}{c}\text { Halicyclops bowmani Rocha \& } \\
\text { Iliffe, } 1993\end{array}$ & $\mathrm{~B}, \mathrm{e}$ & $\mathrm{W}$ & 2 & & 89 & 21 & & $x$ & $x$ & $x$ & & [38] \\
\hline $\begin{array}{l}\text { Halicyclops herbsti Rocha \& } \\
\text { Iliffe, } 1993\end{array}$ & $\mathrm{~B}, \mathrm{e}$ & $\mathrm{W}$ & 1 & & 89 & 21 & & $x$ & $x$ & $x$ & & \\
\hline $\begin{array}{l}\text { Halicyclops ytororoma Lotufo \& } \\
\text { Rocha, } 1993\end{array}$ & $\mathrm{P}$ & $\mathrm{W}$ & 2 & & 89 & 21 & & $x$ & $x$ & $x$ & & [39] \\
\hline $\begin{array}{c}\text { Speleoithona bermudensis Rocha } \\
\text { \& Iliffe, } 1993\end{array}$ & $\mathrm{~B}, \mathrm{e}$ & $\mathrm{W}$ & 2 & & 3 & 2 & & $x$ & & & $\mathrm{Cr}$ & [38] \\
\hline \multicolumn{13}{|l|}{ Copepoda, Harpacticoida } \\
\hline $\begin{array}{c}\text { Intercrusia problematica } \\
\text { Huys, } 1996\end{array}$ & $\mathrm{~B}, \mathrm{e}$ & $\mathrm{W}$ & 1 & & 2 & 1 & & & & $x$ & & [40] \\
\hline $\begin{array}{l}\text { Neoechinophora fosshageni } \\
\text { Huys, } 1996\end{array}$ & $\mathrm{~B}, \mathrm{e}$ & $\mathrm{W}$ & 2 & & 5 & 4 & & $x$ & $x$ & $x$ & & [40] \\
\hline $\begin{array}{l}\text { Neoechinophora daltonae } \\
\text { Huys, } 1996\end{array}$ & $\mathrm{~B}, \mathrm{e}$ & $\mathrm{W}$ & 3 & & 5 & 4 & & $x$ & $x$ & $x$ & & [40] \\
\hline $\begin{array}{c}\text { Superornatiremis mysticus } \\
\text { Huys, } 1996\end{array}$ & $\mathrm{~B}, \mathrm{e}$ & $\mathrm{W}$ & 3 & & 2 & 1 & & & & $x$ & & [40] \\
\hline \multicolumn{13}{|l|}{ Copepoda, Misophrioida } \\
\hline $\begin{array}{c}\text { Speleophria bivexilla Boxshall \& } \\
\text { Iliffe, } 1986\end{array}$ & $\mathrm{~B}, \mathrm{e}$ & $\mathrm{W}$ & 1 & & 6 & 5 & & $x$ & $x$ & $x$ & $\mathrm{Cr}$ & [41] \\
\hline $\begin{array}{l}\text { Speleophriopsis scottodicarloi } \\
\text { (Boxshall \& Iliffe, 1990) }\end{array}$ & $\mathrm{B}, \mathrm{e}$ & $\mathrm{W}$ & 2 & & 5 & 4 & & & $x$ & $x$ & $\mathrm{Cr}$ & [42] \\
\hline \multicolumn{13}{|l|}{ Copepoda, Platycopioida } \\
\hline $\begin{array}{c}\text { Antrisocopia prehensilis } \\
\text { Fosshagen in Fosshagen \& } \\
\text { Iliffe, } 1985\end{array}$ & $\mathrm{~B}, \mathrm{e}$ & $\mathrm{W}$ & 1 & G & 1 & 1 & & $x$ & & $x$ & $\mathrm{Cr}$ & [34] \\
\hline $\begin{array}{l}\text { Nanocopia minuta Fosshagen } \\
\text { in Fosshagen \& Iliffe, } 1988\end{array}$ & $\mathrm{~B}, \mathrm{e}$ & $\mathrm{W}$ & 1 & G & 1 & 1 & & $x$ & & $x$ & $\mathrm{Cr}$ & [43] \\
\hline
\end{tabular}


Table 1. Cont.

\begin{tabular}{|c|c|c|c|c|c|c|c|c|c|c|c|c|}
\hline & \multirow{2}{*}{ Ecology } & \multirow{2}{*}{$\begin{array}{l}\text { Species } \\
\text { Habitat }\end{array}$} & \multirow{2}{*}{$\begin{array}{l}\text { \# Bermuda } \\
\text { Caves }\end{array}$} & \multirow{2}{*}{$\begin{array}{l}\text { Monospecific } \\
\text { Taxa }\end{array}$} & \multirow{2}{*}{$\begin{array}{l}\text { Species in } \\
\text { the Genus }\end{array}$} & \multicolumn{2}{|c|}{ Habitat Relationship } & \multicolumn{3}{|c|}{ Region Relationship } & \multirow{2}{*}{$\begin{array}{l}\text { Conservation } \\
\text { Status }\end{array}$} & \multirow{2}{*}{ Reference } \\
\hline & & & & & & Cave/Interstitial & Deep Sea & Caribbean & Atlantic & Other & & \\
\hline \multicolumn{13}{|l|}{ Ostracoda, Bairdiidae } \\
\hline $\begin{array}{l}\text { Aponesidea iliffei Maddocks in } \\
\text { Maddocks \& Iliffe, } 1986\end{array}$ & $\mathrm{~B}, \mathrm{e}$ & $\mathrm{S}$ & 10 & & 4 & & & & & $x$ & & [44] \\
\hline $\begin{array}{l}\text { Havanardia keiji Maddocks in } \\
\text { Maddocks \& Iliffe, } 1986\end{array}$ & $\mathrm{P}, \mathrm{e}$ & $\mathrm{S}$ & 9 & & 12 & & & $x$ & $x$ & & & [44] \\
\hline $\begin{array}{c}\text { Neonesidea omnivaga } \\
\text { Maddocks in Maddocks \& } \\
\text { Iliffe, } 1986\end{array}$ & $\mathrm{P}, \mathrm{e}$ & $\mathrm{S}$ & 3 & & 99 & & & $x$ & $x$ & $x$ & & [44] \\
\hline $\begin{array}{l}\text { Paranesidea sterreri Maddocks } \\
\text { in Maddocks \& Iliffe, } 1986\end{array}$ & $\mathrm{P}, \mathrm{e}$ & S & 2 & & 41 & & & $x$ & $x$ & $x$ & & [44] \\
\hline \multicolumn{13}{|l|}{ Ostracoda, Candonidae } \\
\hline $\begin{array}{l}\text { Dolerocypria bifurca Maddocks } \\
\text { in Maddocks \& Iliffe, } 1986\end{array}$ & $\mathrm{~B}, \mathrm{e}$ & $\mathrm{S}$ & 3 & & 14 & 2 & & & & $x$ & & [44] \\
\hline $\begin{array}{l}\text { Paracypris crispa Maddocks in } \\
\text { Maddocks \& Iliffe, } 1986\end{array}$ & $\mathrm{P}, \mathrm{e}$ & $\mathrm{S}$ & 3 & & 39 & & & $x$ & $x$ & $x$ & & [44] \\
\hline \multicolumn{13}{|l|}{ Ostracoda, Cytherellidae } \\
\hline $\begin{array}{l}\text { Cytherella kornickeri Maddocks } \\
\text { in Maddocks \& Iliffe, } 1986\end{array}$ & $\mathrm{~B}, \mathrm{e}$ & $\mathrm{S}$ & 6 & & 158 & & + & $x$ & $x$ & $x$ & & [44] \\
\hline \multicolumn{13}{|l|}{$\begin{array}{c}\text { Ostracoda, } \\
\text { Cylindroleberididae }\end{array}$} \\
\hline $\begin{array}{l}\text { Parasterope muelleri } \\
\text { (Skogsberg, 1920) }\end{array}$ & $\mathrm{P}$ & & 2 & & 46 & & & & $x$ & $x$ & & [45] \\
\hline \multicolumn{13}{|l|}{ Ostracoda, Deeveyidae } \\
\hline $\begin{array}{c}\text { Spelaeoecia bermudensis Angel } \\
\text { \& Iliffe, } 1987\end{array}$ & $\mathrm{~B}, \mathrm{e}$ & $\mathrm{W}$ & 11 & & 11 & 10 & & $x$ & & & $\mathrm{Cr}$ & [46] \\
\hline
\end{tabular}


Table 1. Cont.

\begin{tabular}{|c|c|c|c|c|c|c|c|c|c|c|c|c|}
\hline & \multirow{2}{*}{ Ecology } & \multirow{2}{*}{$\begin{array}{l}\text { Species } \\
\text { Habitat }\end{array}$} & \multirow{2}{*}{$\begin{array}{l}\text { \# Bermuda } \\
\text { Caves }\end{array}$} & \multirow{2}{*}{$\begin{array}{l}\text { Monospecific } \\
\text { Taxa }\end{array}$} & \multirow{2}{*}{$\begin{array}{l}\text { Species in } \\
\text { the Genus }\end{array}$} & \multicolumn{2}{|c|}{ Habitat Relationship } & \multicolumn{3}{|c|}{ Region Relationship } & \multirow{2}{*}{$\begin{array}{l}\text { Conservation } \\
\text { Status }\end{array}$} & \multirow{2}{*}{ Reference } \\
\hline & & & & & & Cave/Interstitial & Deep Sea & Caribbean & Atlantic & Other & & \\
\hline \multicolumn{13}{|l|}{ Ostracoda, Halocypridae } \\
\hline $\begin{array}{c}\text { Metapolycope duplex Kornicker } \\
\text { \& Iliffe, } 1989\end{array}$ & $\mathrm{~B}, \mathrm{e}$ & $\mathrm{S}$ & 12 & & 10 & & 4 & & $x$ & & & {$[47]$} \\
\hline $\begin{array}{l}\text { Micropolycope eurax Kornicker } \\
\text { \& Iliffe, } 1989\end{array}$ & $\mathrm{~B}, \mathrm{e}$ & $\mathrm{S}$ & 3 & & 11 & 1 & & & $x$ & $x$ & & {$[47]$} \\
\hline $\begin{array}{l}\text { Micropolycope styx Kornicker } \\
\text { \& Iliffe, } 1989\end{array}$ & $\mathrm{~B}, \mathrm{e}$ & $\mathrm{S}$ & 6 & & 11 & 1 & & & $x$ & $x$ & & [47] \\
\hline \multicolumn{13}{|l|}{ Ostracoda, Philomedidae } \\
\hline $\begin{array}{l}\text { Pseudophilomedes kylix } \\
\text { Kornicker \& Iliffe, } 1989\end{array}$ & $\mathrm{~B}, \mathrm{e}$ & $\mathrm{S}$ & 5 & & 11 & & & & $x$ & $x$ & & [47] \\
\hline \multicolumn{13}{|l|}{ Ostracoda, Polycopidae } \\
\hline $\begin{array}{c}\text { Polycopissa anax Kornicker \& } \\
\text { Iliffe, } 1989\end{array}$ & $\mathrm{~B}, \mathrm{e}$ & $\mathrm{S}$ & 8 & & 4 & & & & $x$ & $x$ & & [47] \\
\hline \multicolumn{13}{|l|}{ Ostracoda, Pontocyprididae } \\
\hline $\begin{array}{c}\text { Iliffeoecia iliffei } \\
\text { Maddocks, } 1991\end{array}$ & B & S & 2 & & 3 & & & & & $x$ & & {$[48]$} \\
\hline $\begin{array}{l}\text { Kareloecia minacis (Maddocks } \\
\text { in Maddocks \& Iliffe, 1986) }\end{array}$ & $\mathrm{B}, \mathrm{e}$ & $\mathrm{S}$ & 1 & & 2 & 1 & & & & $x$ & & [44] \\
\hline $\begin{array}{c}\text { Thomontocypris lurida } \\
\text { (Maddocks in Maddocks \& } \\
\text { Iliffe, 1986) }\end{array}$ & $\mathrm{B}, \mathrm{e}$ & $\mathrm{S}$ & 5 & & 9 & & 6 & $x$ & $x$ & $x$ & & [44] \\
\hline \multicolumn{13}{|l|}{ Ostracoda, Sarsiellidae } \\
\hline $\begin{array}{l}\text { Eusarsiella styx Kornicker \& } \\
\text { Iliffe, } 1989\end{array}$ & $\mathrm{~B}, \mathrm{e}$ & $\mathrm{S}$ & 6 & & 86 & 7 & + & $x$ & $x$ & $x$ & & [47] \\
\hline \multicolumn{13}{|l|}{ Caridea, Atyidae } \\
\hline $\begin{array}{l}\text { Typhlatya iliffei Hart \& } \\
\text { Manning, } 1981\end{array}$ & $\mathrm{~B}, \mathrm{e}$ & $\mathrm{W}$ & 1 & & 17 & 16 & & $x$ & $x$ & $x$ & $\mathrm{Cr}$ & [19] \\
\hline \multicolumn{13}{|l|}{ Caridea, Alpheidae } \\
\hline $\begin{array}{l}\text { Bermudacaris harti Anker \& } \\
\text { Iliffe, } 2000\end{array}$ & $\mathrm{~B}, \mathrm{e}$ & $\mathrm{W}$ & 4 & & 3 & & & & & $x$ & & [20] \\
\hline
\end{tabular}


Table 1. Cont.

\begin{tabular}{|c|c|c|c|c|c|c|c|c|c|c|c|c|}
\hline & \multirow{2}{*}{ Ecology } & \multirow{2}{*}{$\begin{array}{l}\text { Species } \\
\text { Habitat }\end{array}$} & \multirow{2}{*}{$\begin{array}{l}\text { \# Bermuda } \\
\text { Caves }\end{array}$} & \multirow{2}{*}{$\begin{array}{l}\text { Monospecific } \\
\text { Taxa }\end{array}$} & \multirow{2}{*}{$\begin{array}{l}\text { Species in } \\
\text { the Genus }\end{array}$} & \multicolumn{2}{|c|}{ Habitat Relationship } & \multicolumn{3}{|c|}{ Region Relationship } & \multirow{2}{*}{$\begin{array}{l}\text { Conservation } \\
\text { Status }\end{array}$} & \multirow{2}{*}{ Reference } \\
\hline & & & & & & Cave/Interstitial & Deep Sea & Caribbean & Atlantic & Other & & \\
\hline \multicolumn{13}{|l|}{ Caridea, Barbouriidae } \\
\hline $\begin{array}{l}\text { Barbouria cubensis (von } \\
\quad \text { Martens, 1872) }\end{array}$ & B & W & 3 & G & 1 & 8 & & $x$ & $x$ & $x$ & $\mathrm{Cr}$ & [22] \\
\hline $\begin{array}{l}\text { Parhippolyte sterreri (Hart \& } \\
\text { Manning, 1981) }\end{array}$ & B & W & 2 & & 6 & 5 & & $x$ & $x$ & $\mathrm{X}$ & $\mathrm{Cr}$ & [19] \\
\hline \multicolumn{13}{|l|}{ Caridea, Procarididae } \\
\hline $\begin{array}{l}\text { Procaris chacei Hart \& } \\
\text { Manning, } 1986\end{array}$ & $\mathrm{~B}, \mathrm{e}$ & W & 1 & & 5 & 4 & & $x$ & $x$ & $x$ & $\mathrm{Cr}$ & [49] \\
\hline \multicolumn{13}{|l|}{ Isopoda, Atlantasellidae } \\
\hline $\begin{array}{c}\text { Atlantasellus cavernicolus } \\
\text { Sket, } 1979\end{array}$ & $\mathrm{~B}, \mathrm{e}$ & $\mathrm{S}$ & 1 & & 2 & 1 & 1 & & $x$ & & $\mathrm{Cr}$ & {$[50]$} \\
\hline \multicolumn{13}{|l|}{ Isopoda, Cirolanidae } \\
\hline $\begin{array}{l}\text { Arubolana aruboides (Bowman } \\
\text { \& Iliffe, 1983) }\end{array}$ & $\mathrm{B}, \mathrm{e}$ & W & 4 & & 4 & 3 & & $x$ & & $x$ & $\mathrm{Cr}$ & [51] \\
\hline \multicolumn{13}{|l|}{ Isopoda, Leptanthuridae } \\
\hline \multicolumn{13}{|l|}{ Isopoda, Stenetriidae } \\
\hline $\begin{array}{l}\text { Stenobermuda iliffei } \\
\text { Kensley, } 1994\end{array}$ & $\mathrm{~B}, \mathrm{e}$ & $\mathrm{S}$ & 1 & & 6 & & & $x$ & $x$ & $x$ & & [53] \\
\hline \multicolumn{13}{|l|}{ Amphipoda, Amphilochidae } \\
\hline $\begin{array}{l}\text { Hourstonius petulans } \\
\text { (Karaman, 1980) }\end{array}$ & $\mathrm{P}$ & S & 1 & & 18 & & 1 & & & $x$ & & [54] \\
\hline \multicolumn{13}{|l|}{ Amphipoda, Bogidiellidae } \\
\hline $\begin{array}{l}\text { Bermudagidiella bermudiensis } \\
\text { (Stock, Sket \& Iliffe, 1987) }\end{array}$ & $\mathrm{B}, \mathrm{e}$ & S & 2 & G & 1 & 115 & 114 & $x$ & $x$ & $x$ & $\mathrm{Cr}$ & [55] \\
\hline \multicolumn{13}{|l|}{ Amphipoda, Liljeborgiidae } \\
\hline Idunella sketi Karaman, 1980 & $\mathrm{P}$ & $\mathrm{S}$ & 1 & & 44 & 1 & & $x$ & $x$ & $x$ & $\mathrm{Cr}$ & [56] \\
\hline $\begin{array}{c}\text { Idunella verrilli Yabut \& } \\
\text { Sawicki, } 2015\end{array}$ & $\mathrm{~B}, \mathrm{e}$ & & 1 & & 44 & 1 & & $x$ & $x$ & $x$ & & [56] \\
\hline
\end{tabular}


Table 1. Cont.

\begin{tabular}{|c|c|c|c|c|c|c|c|c|c|c|c|c|}
\hline & \multirow{2}{*}{ Ecology } & \multirow{2}{*}{$\begin{array}{l}\text { Species } \\
\text { Habitat }\end{array}$} & \multirow{2}{*}{$\begin{array}{l}\text { \# Bermuda } \\
\text { Caves }\end{array}$} & \multirow{2}{*}{$\begin{array}{l}\text { Monospecific } \\
\text { Taxa }\end{array}$} & \multirow{2}{*}{$\begin{array}{l}\text { Species in } \\
\text { the Genus }\end{array}$} & \multicolumn{2}{|c|}{ Habitat Relationship } & \multicolumn{3}{|c|}{ Region Relationship } & \multirow{2}{*}{$\begin{array}{l}\text { Conservation } \\
\text { Status }\end{array}$} & \multirow{2}{*}{ Reference } \\
\hline & & & & & & Cave/Interstitial & Deep Sea & Caribbean & Atlantic & Other & & \\
\hline \multicolumn{13}{|l|}{$\begin{array}{c}\text { Amphipoda, } \\
\text { Phoxocephalidae }\end{array}$} \\
\hline \multicolumn{13}{|l|}{ Amphipoda, Podoceridae } \\
\hline $\begin{array}{l}\text { Podobothrus bermudensis } \\
\text { Barnard \& Clark, } 1985\end{array}$ & $\mathrm{~B}, \mathrm{e}$ & & 1 & G & 1 & & + & $x$ & $x$ & $x$ & & [58] \\
\hline \multicolumn{13}{|l|}{$\begin{array}{c}\text { Amphipoda, } \\
\text { Pseudoniphargidae }\end{array}$} \\
\hline $\begin{array}{c}\text { Pseudoniphargus grandimanus } \\
\text { Stock, Holsinger, Sket \& } \\
\text { Iliffe, } 1986\end{array}$ & $\mathrm{~B}, \mathrm{e}$ & W & 20 & & 72 & 71 & & & $x$ & $x$ & $\mathrm{Cr}$ & [59] \\
\hline \multicolumn{13}{|l|}{ Ingolfiellida, Ingolfiellidae } \\
\hline $\begin{array}{l}\text { Ingolfiella (Tethydiella) longipes } \\
\text { Stock, Sket \& Iliffe, } 1987\end{array}$ & $\mathrm{~B}, \mathrm{e}$ & S & 1 & & 44 & + & + & $x$ & $x$ & $x$ & $\mathrm{Cr}$ & [55] \\
\hline \multicolumn{13}{|l|}{ Tanaidacea, Apseudidae } \\
\hline $\begin{array}{l}\text { Paradoxapseudes bermudeus } \\
\text { (Băcescu, 1980) }\end{array}$ & $\mathrm{P}$ & S & 1 & & 18 & 3 & 1 & $x$ & & $x$ & & [61] \\
\hline \multicolumn{13}{|l|}{ Mictacea, Mictocarididae } \\
\hline $\begin{array}{l}\text { Mictocaris halope Bowman \& } \\
\text { Iliffe, } 1985\end{array}$ & $\mathrm{~B}, \mathrm{e}$ & $\mathrm{W}$ & 4 & $\mathrm{O}$ & 1 & 3 & 3 & $x$ & & $x$ & $\mathrm{Cr}$ & [21] \\
\hline \multicolumn{13}{|l|}{ Cumacea, Nannastacidae } \\
\hline $\begin{array}{l}\text { Cumella (Cumella) iliffei } \\
\text { Băcescu, } 1992\end{array}$ & $\mathrm{~B}, \mathrm{e}$ & $\mathrm{S}$ & 2 & & 102 & 15 & & $x$ & $x$ & $x$ & & [62] \\
\hline $\begin{array}{c}\text { Cumella (Cumella) ocellata } \\
\text { Băcescu, } 1992\end{array}$ & $\mathrm{~B}, \mathrm{e}$ & S & 1 & & 102 & 15 & & $X$ & $x$ & $x$ & & [62] \\
\hline $\begin{array}{l}\text { Cumella (Cumella) spinosa } \\
\text { Băcescu \& Iliffe, } 1991\end{array}$ & & $S$ & 1 & & 102 & 15 & & $x$ & $x$ & $x$ & & [63] \\
\hline
\end{tabular}


Table 1. Cont.

\begin{tabular}{|c|c|c|c|c|c|c|c|c|c|c|c|c|}
\hline & \multirow{2}{*}{ Ecology } & \multirow{2}{*}{$\begin{array}{l}\text { Species } \\
\text { Habitat }\end{array}$} & \multirow{2}{*}{$\begin{array}{l}\text { \# Bermuda } \\
\text { Caves }\end{array}$} & \multirow{2}{*}{$\begin{array}{l}\text { Monospecific } \\
\text { Taxa }\end{array}$} & \multirow{2}{*}{$\begin{array}{l}\text { Species in } \\
\text { the Genus }\end{array}$} & \multicolumn{2}{|c|}{ Habitat Relationship } & \multicolumn{3}{|c|}{ Region Relationship } & \multirow{2}{*}{$\begin{array}{l}\text { Conservation } \\
\text { Status }\end{array}$} & \multirow{2}{*}{ Reference } \\
\hline & & & & & & Cave/Interstitial & Deep Sea & Caribbean & Atlantic & Other & & \\
\hline \multicolumn{13}{|l|}{ Cumacea, Nannastacidae } \\
\hline $\begin{array}{l}\text { Schizotrema agglutinanta } \\
\text { (Băcescu, 1971) }\end{array}$ & $\mathrm{P}$ & S & 8 & & 20 & 1 & & $x$ & $x$ & $x$ & & [64] \\
\hline $\begin{array}{l}\text { Schizotrema wittmanni } \\
\text { Petrescu \& Sterrer, } 2001\end{array}$ & $\mathrm{P}$ & S & 1 & & 20 & 1 & & $x$ & $x$ & $x$ & & [65] \\
\hline \multicolumn{13}{|l|}{ Mysida, Mysidae } \\
\hline $\begin{array}{l}\text { Bermudamysis speluncola } \\
\text { Băcescu \& Iliffe, } 1986\end{array}$ & $\mathrm{~B}, \mathrm{e}$ & $\mathrm{W}$ & 2 & G & 1 & 2 & 1 & & $x$ & $x$ & $\mathrm{Cr}$ & [66] \\
\hline $\begin{array}{c}\text { Platyops sterreri Băcescu \& } \\
\text { Iliffe, } 1986\end{array}$ & $\mathrm{~B}, \mathrm{e}$ & $\mathrm{W}$ & 2 & G & 1 & 2 & 1 & & $x$ & $x$ & $\mathrm{Cr}$ & [66] \\
\hline
\end{tabular}


Sixty-three species are stygobionts, and fifteen stygophiles (i.e., aquatic animals living in and outside caves). Most of Bermuda's endemic cave species have an extremely restricted distribution, with 26 species known only from a single cave, 14 more species limited to just two caves, and 52 species in total occurring in five or fewer caves. The ostracod Iliffeoecia iliffei also occurs in caves from the Galápagos Islands, while the barbouriid shrimp Barbouria cubensis inhabits caves from Bahamas, Caicos Islands, Cayman Islands, Cuba, Jamaica, and Mexico's Yucatan Peninsula, and Parhippolyte sterreri is found from caves in the Bahamas and the Yucatan Peninsula, in addition to Bermuda [67,68]. The stygophile ostracods Havanardia keiji, Neonesidea omnivaga, Paranesidea sterreri, Cytherella bermudensis, and Paracypris crispa were described from sample specimens collected in caves but have been also found in the island's open water environments, and are endemic to Bermuda [44]. The copepods Epacteriscus rapax, Exumella polyarthra, Halicyclops ytororoma, and Ridgewayia marki, the ostracod Parasterope muelleri, the cumaceans Schizotrema agglutinanta and S. wittmanni, the amphipods Hourstonius petulans and Idunella sketi, and the tanaidacean Paradoxapseudes bermudeus are also found from open water or interstitial environments outside Bermuda [32-34,39,65,68-71].

Considering Bermuda's isolated mid-ocean location, biogeographical affinities of the cave species may provide significant clues as to their origins. While the Gulf Stream may have transported some species to the island, others may have survived on submerged or emergent seamounts near the Mid-Atlantic Ridge, originated from Tethyan relicts, or are derived from deep-sea taxa [5]. Here are a few examples: The misophrioid copepod Speleophria bivexilla has congeners inhabiting caves from the Balearic Islands, Croatia, northwestern and southern Australia, and the Yucatan Peninsula, suggesting possible dispersal by plate tectonic processes [72]. The atyid shrimp Typhlatya iliffei belongs to an exclusively cave-adapted genus with species spread around the Caribbean and Western Mediterranean, as well as the Galapagos Islands, Ascension Island, and Madagascar. Their ancestors likely inhabited the ancient Tethys Sea with both vicariance (the opening of the Atlantic) and dispersal leading to their isolation and divergence [73]. The stygobiont amphipod Pseudoniphargus includes species from North Africa, the Mediterranean region and its islands, the Iberian Peninsula, the Canary Islands, Madeira, and the Azores, in addition to two species in Bermuda, on the west side of the mid-Atlantic rift, but is absent from the American continent or from the Caribbean. Based on phylogenetic analyses, the estimated age of the Pseudoniphargus lineage on Bermuda is relatively young, only $5 \mathrm{Ma}$ [74] The ostracod Spelaeoecia bermudensis belongs to a stygobiont genus with seven species from the Bahamas, two from Cuba, and one each from Jamaica, the Yucatan Peninsula, and Bermuda. The cluster of this ostracod in the Bahamas suggests possible ocean dispersal via the Gulf Stream rafting adults or larvae to Bermuda [75].

While numerous cave taxa in Bermuda are significant in their presence, the absence of several globally prominent stygobiont taxa is also remarkable. The crustacean class Remipedia inhabits anchialine caves in the Bahamas (21 spp.), Yucatan Peninsula (3 spp.), the Dominican Republic (1 sp.), Cuba (1 sp.), and Belize (1 sp.), plus the Canary Islands (2 spp.) and Australia (1 sp.) but, despite many hundreds of cave dives, has never been observed in Bermuda $[76,77]$. The ostracod stygobiont genus Humphreysella has a similar distribution and ecology to Remipedia, while being absent from Bermuda. Humphreysella occurs in caves in the Bahamas (3 spp.), Yucatan Peninsula (1 sp.), Cuba (1 sp.), Jamaica (1 sp.), Galápagos Islands (1 sp.), and Canary Islands (1 sp.), as well as having a closely related monotypic genus in Western Australia and Christmas Island (Indian Ocean) [24,78].

Important questions arise as to the age and origins of Bermuda's endemic cave fauna. The Bermuda seamount formed from volcanic eruptions occurring approximately $35 \mathrm{mil}-$ lion years ago. Due to its attachment to the North American Plate, the seamount remained at a constant distance from the North American coast, but an ever-increasing distance from Europe as the Atlantic Ocean expanded. Limestone bedrock containing all known caves is only 1-2 million years in age. The limestone capping the summit of the Bermuda seamount extends down to about $30 \mathrm{~m}$ below present sea level, such that during Pleistocene regressions to $130 \mathrm{~m}$, all of the island's limestone and, thus, its caves, were exposed above 
the ocean and were dry. The exceptionally large stalactites and stalagmites in the now underwater caves, and the level of the Pleistocene ocean, corroborate that caves must have been continuously dry for many tens thousands of years at a time (Figure 2B). It was not until about 7000 years ago that the sea level rise from the last glacial maximum ( 20,000 years ago) caused Bermuda's caves to become flooded [9].

Considering the high diversity, unique ecological and taxonomic nature of the cave fauna, a relatively modern origin by cave colonization from the open ocean is highly unlikely and can be ruled out. Instead, the cave species currently found in Bermuda must have been dark-adapted organisms that moved into caves from suitable crevice or crevicular habitats in the volcanic bedrock or sides of the Bermuda seamount occurring at depths greater than where sea level was during the last glacial maximum. The presence of a highly diverse and endemic stygobiont fauna in a young $(\sim 21,000$ years old $)$ volcanic cave in the Canary Islands presents a comparable case. La Corona lava tube on Lanzarote formed during the last ice age as a dry cave that was flooded by rising sea level to a maximum water depth of $64 \mathrm{~m}$ [79].

\section{Threats to Bermuda's Cave Fauna}

Bermuda represents an extreme case of threats to marine cave fauna and a microcosm of issues facing caves around the world. The primary threats to Bermuda caves and their fauna include: (i) filling and quarrying activities, (ii) water pollution, (iii) dumping and littering, (iv) vandalism [80], and (v) climate change [81]. Since most of Bermuda's endemic stygiobionts inhabit only a single cave or cave system, pollution of these habitats can threaten entire species with extinction.

Population growth and land development have adversely affected Bermuda's caves and cave fauna. The islands have a total land area of $53.3 \mathrm{~km}^{2}$, of which $20 \%$ (or 10 $\mathrm{km}^{2}$ ) is forest and woodland [77]. As of May 2021, Bermuda's population was estimated to be 62,069 , down from a high of 66,257 in 2005 [82], yielding an average population density of 1164 persons per $\mathrm{km}^{2}$. In such a small, densely populated island, considerable human pressures have been brought to bear on the relatively unknown and poorly appreciated caves.

Intentional dumping of large quantities of refuse, raw sewage, and waste fuel oil into anchialine cave pools in Bermuda resulted in the depletion of dissolved oxygen and production of hydrogen sulfide in deep lakes from Government Quarry and Bassett's Caves [83]. The resulting anoxic conditions not only eliminated all typical cave invertebrates, but also formed black metal sulfide precipitates that substantially reduced water clarity. Such polluted waters can move for considerable distances underground and appear years later in distant cave pools. In addition, broken or missing speleothems, graffiti, names on cave walls, and litter are common sights in many dry caves in Bermuda, while sinkholes and cave entrances were long used for trash disposal [80] (Figure 2F). Tidal currents suck floating plastic bottles and bags into coastal caves (Figure 2E). Such negative impacts to caves reduce their esthetic value and, therefore, make it more difficult to justify protective measures.

Climate change is a medium-term to long-term threat, since warming climate causes an increased frequency and strength of hurricanes and sea level rise, which will directly affect the habitat availability. Furthermore, cave organisms are especially vulnerable to rapid environmental changes [81,84].

In an effort to protect caves, Bermuda's planning laws now afford caves the highest level of protection [85]. The Fourth Schedule of the Planning Act 1974 states: "The protection of caves shall take precedence over all other planning considerations and the Board shall refuse any development application or planning of subdivision if, in the opinion of the Board, the proposal will have detrimental impact on a cave entrance or underlying cave." The Protected Species Act 2003 and Protected Species Order 2012 list 22 cave-dwelling species for legal protection, while the 2014 "Management Plan for Bermuda's Critically Endangered Cave Fauna" seeks adequate protection for the entire cave habitat. The Man- 
agement Plan is designed to protect caves through legislation and raised public awareness, comprehensive mapping of caves, identifying and managing point source pollution, monitoring cave air and water, examining the potential for hatchery breeding, facilitating ecological research, and undertaking active restoration of impacted caves [85].

Unfortunately, loopholes in the laws have allowed important caves with endangered and protected species to be destroyed. Wilkinson Quarry Cave at the northern end of the Walsingham Track was discovered during blasting operations in 2002. The cave contained profuse and actively growing speleothems and a large network of submerged cave passages where biological collections identified four species of stygobiont crustaceans on the IUCN Red List. While biology and conservation experts pressed for protection and preservation of the cave, consultants hired by quarry management supported its destruction arguing that the cave was (1) small, (2) structurally compromised and therefore unsafe, and (3) not ecologically or esthetically significant [86]. Despite the Planning Act law and the presence of endangered species, the Bermuda Development Applications Board approved destruction of the cave and removal of all bedrock to level off the lower quarry floor.

Another way to safeguard caves is through private and government owned nature reserves that effectively protect undeveloped woodland, karst topography, extensive cave systems, saltwater ponds, and endemic cave-adapted plants, mosses, and ferns. The 400acre (160 ha) Walsingham Tract includes four adjacent nature reserves: the 23-acre (9.3 ha) Walsingham Trust Nature Reserve, 1.25-acre (0.5 ha) Idwal Hughes Nature Reserve, 12-acre (4.9 ha) Blue Hole Hill Park, and Crystal and Fantasy Caves operated by the Wilkinson Trust as commercial tourist attractions [87]. Since the mid-20th century, sections at the northern and southern ends of the Tract have been lost to quarrying, hotel, and residential development, increasing the importance of these nature reserves as a key conservation and restoration areas.

Thus, conservation actions to protect Bermuda's anchialine cave habitat and its unique stygobiont fauna, especially caves in the Walsingham Tract, are crucial. Further research is needed to understand the biodiversity, species biology, population sizes, and carrying capacity of the ecosystem in relation to diverse human activities.

Author Contributions: Conceptualization, T.M.I.; methodology, T.M.I.; validation, T.M.I. and F.C.-G.; investigation, T.M.I.; resources, T.M.I.; data curation, T.M.I. and F.C.-G.; writing-original draft preparation, T.M.I.; writing-review and editing, T.M.I. and F.C.-G.; visualization, T.M.I. and F.C.-G.; supervision, T.M.I.; project administration, T.M.I.; funding acquisition, T.M.I. All authors have read and agreed to the published version of the manuscript.

Funding: This research was funded by National Science Foundation grants BSR-8215672, and DEB-8001836; NOAA Ocean Exploration NA09OAR4600096; NOAA Office of Undersea Research SWS-87-23; and NOAA International Coral Reef Conservation Program NA06NOS46300-85.

Institutional Review Board Statement: Research involving the collection of live animals was conducted in accordance with and approval from the Government of Bermuda Department of Conservation Services.

Informed Consent Statement: Not applicable.

Data Availability Statement: The data presented in this study is available in the cited references.

Acknowledgments: This paper is dedicated to Wolfgang Sterrer, without whose encouragement and considerable assistance, these submarine cave biology studies in Bermuda would never have been inaugurated. We sincerely acknowledge the substantial help provided by cave divers Rob Power, Paul Hobbs, Dennis Williams, Gil Nolan, Jason and Chrissy Richards, Jill Heinerth, Paul Heinerth, and Brian Kakuk. Also, taxonomists Boris Sket, Thomas Bowman, C.W. Hart Jr., Raymond Manning, Louis Kornicker, Geoff Boxshall, John Holsinger, Mihai Băcescu, Jan Stock, Audun Fosshagen, Modest Gutu, Carlos Rocha, Iorgu Petrescu, Rosalie Maddocks, Lazare Botosaneanu, and others. We also thank the following individuals or organizations in Bermuda: Annie Glasspool, Struan Smith, the Bermuda Biological Station, the Bermuda Aquarium and Zoo, the Bermuda Zoological Society, the Bermuda Underwater Exploration Institute, the Bermuda National Trust, the Walsingham Trust, and many others. 
Conflicts of Interest: The authors declare no conflict of interest. The funders had no role in the design of the study; in the collection, analyses, or interpretation of data; in the writing of the manuscript, or in the decision to publish the results.

\section{References}

1. Smithsonian.com. Bermuda-History and Heritage. Available online: https://www.smithsonianmag.com/travel/bermudahistory-and-heritage-14340790/?no-ist (accessed on 8 June 2021).

2. Iliffe, T.M. Speleological history of Bermuda. Acta Carsologica 1993, 22, 114-135.

3. Lefroy, L.H. Memorials of the Discovery and Early Settlement of the Bermudas or Somers Islands; Franklin Classics Trade Press: London, UK, 1877; pp. 1511-1687.

4. Thomson, C.W. The Voyage of the "Challenger". The Atlantic; A Preliminary Account of the General Results of the Exploring Voyage of H.M.S. "Challenger" during the Year 1873 and the Early Part of the Year 1876; Harper \& Brothers: New York, NY, USA, 1878.

5. Iliffe, T.M.; Hart, C.W.; Manning, R.B. Biogeography and the caves of Bermuda. Nature 1983, 302, 141-142. [CrossRef]

6. Holthuis, L.B. Caridean shrimps found in Land-locked saltwater pools at four Indo-West Pacific localities (Sinai Peninsula, Funafuti Atoll, Maui and Hawaii Islands), with the description of one new genus and four new species. Zool. Verh. 1973, 128, 3-48.

7. Stock, J.H.; Iliffe, T.M.; Williams, D. The concept of "anchialine" reconsidered. Stygologia 1986, 2, 90-92.

8. Bishop, R.E.; Humphreys, W.F.; Cukrov, N.; Žic, V.; Boxshall, G.A.; Cukrov, M.; Iliffe, T.M.; Kršinić, F.; Moore, W.S.; Pohlman, J.W.; et al. 'Anchialine' redefined as a subterranean estuary in a crevicular or cavernous geological setting. J. Crustac. Biol. 2015, 35, 511-514. [CrossRef]

9. van Hengstum, P.J.; Cresswell, J.N.; Milne, G.A.; Iliffe, T.M. Development of anchialine cave habitats and karst subterranean estuaries since the last ice age. Sci. Rep. 2019, 9, 11907. [CrossRef] [PubMed]

10. Rowe, M.P. The Geology of Bermuda; bermudageology.com: Bermuda, UK, 2020.

11. Palmer, A.N.; Palmer, M.V.; Queen, J.M. Geology and origin of the caves of Bermuda. In Proceedings of the Seventh International Congress of Speleology, Sheffield, England, 11-16 September 1977; pp. 336-339.

12. Illife, T.M. The submarine caves of Bermuda. In Proceedings of the Eighth International Congress of Speleology, Bowling Green, KY, USA, 18-24 July 1981; pp. 161-163.

13. Thomas, M.L.H. The Ecology of Harrington Sound, Bermuda; Bermuda Zoological Society: Bermuda, UK, 2005.

14. Iliffe, T.M. Anchialine cave ecology. In Ecosystems of the World. 30. Subterranean Ecosystems; Wilkens, H., Culver, D.C., Humphreys, W.F., Eds.; Elsevier: Amsterdam, The Netherlands, 2000; pp. 59-76.

15. Pohlman, J.W.; Cifuentes, L.A.; Iliffe, T.M. Food Web Dynamics and Biogeochemistry of Anchialine Caves: A Stable Isotope Approach. Ecosyst. World 2000, 30, 351-363.

16. Sket, B.; Iliffe, T.M. Cave fauna of Bermuda. Int. Rev. Gesamten Hydrobiol. Hydrogr. 1980, 65, 871-882. [CrossRef]

17. Culver, D.C.; Sket, B. Hotspots of subterranean biodiversity in caves and wells. J. Cave Karst Stud. 2000, 62, 11-17.

18. Culver, D.C.; Pipan, T. The Biology of Caves and Other Subterranean Habitats; Oxford University Press: Oxford, UK, 2009.

19. Hart, C.W.J.; Manning, R.B. The cavernicolous caridean shrimps of Bermuda (Alpheidae, Hippolytidae, and Atyidae). J. Crustac. Biol. 1981, 1, 441-456. [CrossRef]

20. Anker, A.; Iliffe, T.M. Description of Bermudacaris harti, a new genus, and species (Crustacea: Decapoda: Alpheidae) from anchialine caves of Bermuda. Proc. Biol. Soc. Wash. 2000, 113, 761-775.

21. Bowman, T.E.; Iliffe, T.M. Mictocaris halope, a new unusual peracaridan crustacean from marine caves on Bermuda. J. Crustac. Biol. 1985, 5, 58-73. [CrossRef]

22. von Martens, E. Über Cubanische Crustaceen. Arch. Naturgeschich. 1872, 38, 77-147.

23. IUCN. The IUCN Red List of Threatened Species. Available online: http:/ / www.iucnredlist.org/ (accessed on 30 May 2021).

24. WoRMS. Editorial Board World Register of Marine Species. Available online: http://www.marinespecies.org (accessed on 30 May 2021).

25. Hill, B.F.; Small, E.B.; Iliffe, T.M. Euplotes iliffei n. sp.: A new species of Euplotes (Ciliophora, Hypotrichida) from the marine caves of Bermuda. J. Wash. Acad. Sci. 1986, 76, 244-249.

26. Small, E.B.; Heisler, J.; Sniezek, J.; Iliffe, T.M. Glauconema bermudense n. sp. (Scuticociliatida, Oligohymenophorea), a troglobitic ciliophoran from Bermudian marine caves. Stygologia 1986, 2, 167-179.

27. Sterrer, W.; Iliffe, T.M. Mesonerilla prospera, a new archiannelid from marine caves in Bermuda. Proc. Biol. Soc. Wash. 1982, 95, 509-514.

28. Worsaae, K.; Sterrer, W.; Iliffe, T.M. Longipalpa saltatrix, a new genus and species of the meiofaunal family Nerillidae (Annelida: Polychaeta) from an anchihaline cave in Bermuda. Proc. Biol. Soc. Wash. 2004, 117, 346-362.

29. Erséus, C. A new species of Phallodrilus (Oligochaeta, Tubificidae) from a limestone cave on Bermuda. Sarsia 1986, 71, 7-9. [CrossRef]

30. Moolenbeek, R.; Faber, M.; Iliffe, T.M. Two new species of the genus Caecum (Gastropoda) from marine caves on Bermuda. Stud. Honour Pieter Wagenaar Hummelinck 1988, 123, 209-216.

31. Bartsch, I.; Iliffe, T.M. The halacarid fauna (Halacaridae, Acari) of Bermuda's cave. Stygologia 1985, 1, 300-321. 
32. Fosshagen, A.; Boxshall, G.A.; Iliffe, T.M. The Epacteriscidae, a cave-living family of calanoid copepods. Sarsia 2001, 86, 245-318. [CrossRef]

33. Fosshagen, A. A new genus and species of bottom living calanoid (Copepoda) from Florida and Colombia. Sarsia 1973, 52, 145-154. [CrossRef]

34. Fosshagen, A.; Iliffe, T.M. Two new genera of Calanoida and a new order of Copepoda, Platycopioida, from marine caves on Bermuda. Sarsia 1985, 70, 345-358. [CrossRef]

35. Fosshagen, A. Marine Biological Investigations in the Bahamas. 15. Ridgewayia (Copepoda, Calanoida) and two new genera of calanoids from the Bahamas. Sarsia 1970, 44, 25-58. [CrossRef]

36. Yeatman, H.C. Miostephos leamingtonensis, a new species of copepod from Bermuda. J. Tenn. Acad. Sci. 1980, 55, 21-22.

37. Esterly, C.O. Calanoid Copepoda from the Bermuda Islands. Proc. Am. Acad. Arts Sci. 1911, 47, 219. [CrossRef]

38. Rocha, C.E.F.; Iliffe, T.M. New cyclopoids (Copepoda) from anchialine caves in Bermuda. Sarsia 1993, 78, 43-56. [CrossRef]

39. Lotufo, G.R.; da Rocha, C.E.F. Intertidal interstitial Halicyclops from the Brazilian coast (Copepoda: Cyclopoida). Hydrobiologia 1993, 264, 175-184. [CrossRef]

40. Huys, R. Superornatiremidae fam. nov. (Copepoda: Harpacticoida): An enigmatic family from North Atlantic anchihaline caves. Sci. Mar. 1996, 60, 497-542.

41. Boxshall, G.A.; Iliffe, T.M. New cave-dwelling misophrioids (Crustacea: Copepoda) from Bermuda. Sarsia 1986, 71, 55-64. [CrossRef]

42. Boxshall, G.A.; Iliffe, T.M. Three new species of misophrioid copepods from oceanic islands. J. Nat. Hist. 1990, 24, 595-613. [CrossRef]

43. Fosshagen, A.; Iliffe, T.M. A new genus of Platycopioida (Copepoda) from a marine cave on Bermuda. In Biology of Copepods; Boxshall, G.A., Schminke, H.K., Eds.; Springer: Dordrecht, The Netherlands, 1988; pp. 357-361.

44. Maddocks, R.F.; Iliffe, T.M. Podocopid Ostracoda of Bermudian caves. Stygologia 1986, 2, 26-76.

45. Skogsberg, T. Studies on marine ostracods. Part 1. (Cypridinids, Halocyprids and Polycopids). Zool. Bidr. Fran Upps. 1920, 1-784. [CrossRef]

46. Angel, M.V.; Iliffe, T.M. Spelaeoecia bermudensis, new genus, new species, a halocyprid ostracod from marine caves in Bermuda. J. Crustac. Biol. 1987, 7, 541-553. [CrossRef]

47. Kornicker, L.S.; Iliffe, T.M. Ostracoda (Myodocopina, Cladocopina, Halocypridina) from anchialine caves in Bermuda. Smithson. Contrib. Zool. 1989, 475, 1-88. [CrossRef]

48. Maddocks, R.F. Revision of the family Pontocyprididae (Ostracoda), with new anchialine species and genera from Galapagos Islands. Zool. J. Linn. Soc. 1991, 103, 309-333. [CrossRef]

49. Hart, C.W.J.; Manning, R.B. Two new shrimps (Procarididae and Agostocarididae, new family) from marine caves of the western North Atlantic. J. Crustac. Biol. 1986, 6, 408-416. [CrossRef]

50. Sket, B. Atlantasellus cavernicolus n. gen., n. sp. (Isopoda Asellota, Atlantasellidae n. fam.) from Bermuda. Biol. Vestn. Ljubl. 1979, 7, 175-183.

51. Bowman, T.E.; Iliffe, T.M. Bermudalana aruboides, a new genus and specioes of troglobitic isopoda (Cirolanidae) from marine caves on Bermuda. Proc. Biol. Soc. Wash. 1983, 96, 291-300.

52. Wägele, J.W.; Brandt, A. New west Atlantic localities for the stygobiont paranthurid Curassanthura (Crustacea, Isopoda, Anthuridea) with description of C. bermudensis n. sp. Bijdr. Dierkd. 1985, 55, 324-330. [CrossRef]

53. Kensley, B. Records of shallow-water marine isopods from Bermuda with descriptions of four new species. J. Crustac. Biol. 1994, 14, 319-336. [CrossRef]

54. Karaman, G.S. Revision of the genus Gitanopsis Sars 1895 with description of new genera Afrogitanopsis and Rostrogitanopsis n. gen. (fam. Amphilochidae). Poljopr. Sumar. 1980, 26, 43-69.

55. Stock, J.H.; Sket, B.; Iliffe, T.M. Two new amphipod crustaceans from anchihaline caves in Bermuda. Crustaceana 1987, 53, 54-66. [CrossRef]

56. Karaman, G.S. Revision of genus Idunella Sars with description of new species, I. sketi, n. sp. (Fam. Liljeborgiidae). Acta Adriat. 1980, 21, 409-435.

57. Karaman, G.S. Cocoharpinia iliffei, new genus and species from Bermuda, with remarks to other genera and species (Fam. Phoxocephalidae). (Contribution to the Knowledge of the Amphipoda 103). Stud. Mar. 1980, 9-10, 149-175.

58. Barnard, J.L.; Clark, J. A new sea-cave amphipod from Bermuda (Dulichiidae). Proc. Biol. Soc. Wash. 1985, 98, $1048-1053$.

59. Stock, J.H.; Holsinger, J.R.; Sket, B.; Iliffe, T.M. Two new species of Pseudoniphargus (Amphipoda), in Bermudian groundwaters. Zool. Scr. 1986, 15, 237-249. [CrossRef]

60. Gutu, M.; Iliffe, T.M. Apseudes orghidani, a new species of Tanaidacea (Crustacea) from an anchialine cave on Bermuda. Trav. Mus. Natl. d'Hist. Nat. Grigore Antipa 1989, 30, 161-167.

61. Băcescu, M. Apseudes bermudeus n.sp. from caves around Bermude [sic] Islands [Apseudes bermudeus n.sp. iz pecina oko Bermudskih Otoka]. Acta Adriat. 1980, 21, 401-407.

62. Bäcescu, M. Deux espèces nouvelles de Cumella (Crustacea, Cumacea) des grottes sous-marines de Bermuda. Trav. Mus. Natl. d'Hist. Nat. Grigore Antipa 1992, 32, 257-262.

63. Băcescu, M.; Iliffe, T.M. Nouvelles espèces de Cumella des grottes sous-marines de Bermude. Rev. Behav. Almyracuma Prox. 1991, $36,9-13$. 
64. Băcescu, M. New Cumacea from the littoral waters of Florida (Caribbean Sea). Trav. Mus. Natl. d'Hist. Nat. Grigore Antipa 1971, 11, 5-24.

65. Petrescu, I.; Sterrer, W. Cumacea (Crustacea) from shallow waters of Bermuda. Ann. Nat. Naturhist. Mus. Wien Ser. B Bot. Zool. 2001, 103, 89-128.

66. Băcescu, M.; Iliffe, T.M. Bermudamysis g.n., Platyops g.n. and other mysids from Bermudian caves. Stygologia 1986, 2, 93-104.

67. Ditter, R.E.; Mejía-Ortíz, L.M.; Bracken-Grissom, H.D. Anchialine adjustments: An updated phylogeny and classification for the family Barbouriidae Christoffersen, 1987 (Decapoda: Caridea). J. Crustac. Biol. 2020, 40, 401-411. [CrossRef]

68. Keyser, D.; Schöning, C. Holocene ostracoda (Crustacea) from Bermuda. Senckenberg. Lethaea 2000, 80, 567-591. [CrossRef]

69. Martín, A.; Díaz Díaz, Y.J. Biodiversidad de crustáceos peracáridos en el delta del Río Orinoco, Venezuela. Rev. Biol. Trop. 2006, 55. [CrossRef]

70. Stock, J.H.; Vonk, R. Marine interstitial Amphipoda and Isopoda (Crustacea) from Santiago, Cape Verde Islands. Bijdr. Dierkd. 1992, 62, 21-36. [CrossRef]

71. Winfield, I.; Abarca-Ávila, M.; Ortiz, M.; Cházaro-Olvera, S.; Lozano-Aburto, M.Á. Biodiversidad de los tanaidáceos (Crustacea: Peracarida: Tanaidacea) del Parque Nacional Arrecife Puerto Morelos, Quintana Roo, México. Rev. Mex. Biodivers. 2017, 88, 572-578. [CrossRef]

72. Suárez-Morales, E.; Cervantes-Martínez, A.; Gutiérrez-Aguirre, M.A.; Iliffe, T.M. A new Speleophria (Copepoda, Misophrioida) from an anchialine cave of the Yucatán Peninsula with comments on the biogeography of the genus. Bull. Mar. Sci. 2017, 93, 1-16. [CrossRef]

73. Jurado-Rivera, J.A.; Pons, J.; Alvarez, F.; Botello, A.; Humphreys, W.F.; Page, T.J.; Iliffe, T.M.; Willassen, E.; Meland, K.; Juan, C.; et al. Phylogenetic evidence that both ancient vicariance and dispersal have contributed to the biogeographic patterns of anchialine cave shrimps. Sci. Rep. 2017, 7, 2852. [CrossRef]

74. Stokkan, M.; Jurado-Rivera, J.A.; Oromí, P.; Juan, C.; Jaume, D.; Pons, J. Species delimitation and mitogenome phylogenetics in the subterranean genus Pseudoniphargus (Crustacea: Amphipoda). Mol. Phylogenet. Evol. 2018, 127, 988-999. [CrossRef]

75. Kornicker, L.S.; Iliffe, T.M.; Harrison-Nelson, E. Ostracoda (Myodocopa) from Anchialine Caves and Ocean Blue Holes. Zootaxa 2007, 1565, 1-151. [CrossRef]

76. Koenemann, S.; Iliffe, T.M. Class Remipedia Yager, 1981. In Treatise on Zoology—Anatomy, Taxonomy, Biology: The Crustacea; von Vaupel Klein, J.C., Charmantier-Daures, M., Schram, F.R., Eds.; Brill: Leiden, The Netherlands, 2013; pp. $125-177$.

77. Ballou, L.; Iliffe, T.M.; Kakuk, B.; Gonzalez, B.C.; Osborn, K.J.; Worsaae, K.; Meland, K.; Broad, K.; Bracken-Grissom, H.; Olesen, J. Monsters in the dark: Systematics and biogeography of the stygobitic genus Godzillius (Crustacea: Remipedia) from the Lucayan Archipelago. Eur. J. Taxon. 2021, 751, 115-139. [CrossRef]

78. Moldovan, O.T.; Kováč, L.; Halse, S. Ecological Studies, In Cave Ecology; Springer: Cham, Germany, 2018.

79. Martínez, A.; Gonzalez, B.C.; Núñez, J.; Wilkens, H.; Oromí, P.; Iliffe, T.M.; Worsaae, K. Guide to the Anchialine Ecosystems of Jameos del Agua and Túnel de la Atlántida; Cabildo de Lanzarote: Lanzorote, Spain, 2016.

80. Iliffe, T.M. Bermuda's caves: A non-renewable resource. Environ. Conserv. 1979, 6, 181-186. [CrossRef]

81. Mammola, S.; Cardoso, P.; Culver, D.C.; Deharveng, L.; Ferreira, R.L.; Fišer, C.; Galassi, D.M.P.; Griebler, C.; Halse, S.; Humphreys, W.F.; et al. Scientists' warning on the conservation of subterranean ecosystems. Bioscience 2019, 69, 641-650. [CrossRef]

82. Worldometers.info. Bermuda Population (LIVE). Available online: https://www.worldometers.info/world-population/ bermuda-population/ (accessed on 30 June 2021).

83. Iliffe, T.M.; Jickells, T.D.; Brewer, M.S. Organic pollution of an inland marine cave from Bermuda. Mar. Environ. Res. 1984, 12, 173-189. [CrossRef]

84. Parravicini, V.; Guidetti, P.; Morri, C.; Montefalcone, M.; Donato, M.; Bianchi, C.N. Consequences of sea water temperature anomalies on a Mediterranean submarine cave ecosystem. Estuar. Coast. Shelf Sci. 2010, 86, 276-282. [CrossRef]

85. Glasspool, A. Management Plan for Bermuda's Critically Endangered Cave Fauna; Government of Bermuda, Department of Conservation Services: Bermuda, UK, 2014.

86. Darrell, M. Letter to Bermuda Department of Planning, re: Proposed Demolition of Unstable Mound with Subterranean Void in Wilkinson Quarry, Hamilton Parish. Hamilton: Bermuda. 10 June 2020. Available online: https://www.bnt.bm/images/ Education/WilkinsonQuarryCaveObjection.pdf (accessed on 28 June 2021).

87. Copeland, A. Walsingham Trust Nature Reserve: Special Features and Potential Projects; Museum and Zoo Report Number BAMZ\#3405; Bermuda Aquarium: Bermuda, UK, 2020. 